\title{
Synthesis of glycosylated $\beta^{3}$-homo-threonine conjugates for mucin-like glycopeptide antigen analogues
}

\author{
Florian Karch and Anja Hoffmann-Röder ${ }^{*}$
}

\author{
Full Research Paper \\ Address: \\ Institut für Organische Chemie, Johannes Gutenberg-Universität \\ Mainz, Duesbergweg 10-14, D-55128 Mainz, Germany, Phone: \\ +49-6131-3922417, Fax: +49-6131-3923916 \\ Email: \\ Anja Hoffmann-Röder ${ }^{*}$ - hroeder@uni-mainz.de \\ * Corresponding author \\ Keywords: \\ glycopeptide; glycosylamino acids; $\beta^{3}$-homo-threonine; MUC1 \\ antigens; solid-phase synthesis
}

Open Access

Beilstein J. Org. Chem. 2010, 6, No. 47. doi:10.3762/bjoc.6.47

Received: 08 February 2010

Accepted: 09 April 2010

Published: 12 May 2010

Guest Editor: T. K. Lindhorst

(c) 2010 Karch and Hoffmann-Röder; licensee Beilstein-Institut. License and terms: see end of document.

\begin{abstract}
Glycopeptides from the mucin family decorated with tumour-associated carbohydrate antigens (TACA) have proven to be important target structures for the development of molecularly defined anti-cancer vaccines. The strategic incorporation of $\beta$-amino acid building blocks into such mucin-type sequences offers the potential to create pseudo-glycopeptide antigens with improved bioavailability for tumour immunotherapy. Towards this end, $\mathrm{T}_{\mathrm{N}}$ and TF antigen conjugates $O$-glycosidically linked to Fmoc- $\beta^{3}$ homo-threonine were prepared in good yield via Arndt-Eistert homologation of the corresponding glycosyl $\alpha$-amino acid derivative. By incorporation of $\mathrm{T}_{\mathrm{N}}$-Fmoc- $\beta^{3} \mathrm{hThr}$ conjugate into the 20 amino acid tandem repeat sequence of MUC1 using sequential solid-phase glycopeptide synthesis, a first example of a mixed $\alpha / \beta$-hybrid glycopeptide building block was obtained. The latter is of interest for the development of novel glycoconjugate mimics and model structures for anti-cancer vaccines with increased biological half-life.
\end{abstract}

\section{Introduction}

Glycosylation is the predominant co- and post-translational modification in higher organisms responsible for tailoring and fine-tuning of the activity of proteins involved in fundamental biological recognition events of cell adhesion, cell differentiation and cell growth [1-3]. As a consequence, synthetic oligosaccharides and their conjugates are recognised as important tools for the expanding field of chemical biology [4]. Aberrant glycosylation of cell surface glycoproteins is associated with various pathological incidents, e.g., autoimmune

and infectious diseases and cancer. In the latter case, unusual glycan structures composed of truncated $O$-linked oligosaccharides of carcinoma-derived mucin glycoproteins can be used as markers of the tumourigenic process and as target structures for cancer immunotherapy [5]. Over the last years, mucintype glycopeptides decorated with tumour-associated carbohydrate antigens (TACA) have been shown to trigger strong humoral immunity within molecularly defined vaccine prototypes [6-10]. However, the limited metabolic stability of the 
glycopeptide conjugates represents a major obstacle for the development of efficient carbohydrate-based vaccines. Various strategies towards the incorporation of non-natural hydrolysis resistant carbohydrate analogues into vaccine constructs have been pursued. For instance, stable TACA mimics comprising C-glycosides [11-14], S-glycosides [15-19] and deoxyfluoro sugars [20] have been used to circumvent hydrolytic degradation by endogenous glycosidases.

In principle, antigenicity of the artificial TACA derivatives should be enhanced by minor structural modifications assuming that the conformations remain similar to those of the natural antigens. In this respect, hybrid peptides in which $\beta^{3}$-homoamino acids are used to strategically replace $\alpha$-amino acids might be of interest as platforms for carbohydrate-based vaccines. That is because such mixed $\alpha / \beta$-peptides adopt stable secondary structures closely related to those of natural $\alpha$-peptides $[21,22]$. Moreover, inclusion of a single $\beta$-amino acid into an $\alpha$-peptidic sequence already augments local and/or general stability against proteolytic degradation in vitro and in vivo; thus enabling the development of diverse peptidomimetics for an increasing number of applications [22-25]. Therefore we contemplated the use of mucin-derived $\alpha / \beta$-hybrid glycopeptides as stable mimetics of naturally occurring glycocopeptide antigens for cancer vaccines. We were surprised to see how little precedence was available for this approach. Besides a recent report on $\alpha / \delta$-hybrid peptides derived from Neu2en and L-Glu [26], representing potentially immunogenic mimics of $\alpha$-2,8-linked polysialic acid, only a few $\beta$-glycopeptides comprising $N$-acetylglucosamine [27-29] and $N$-acetylgalactosamine $[30,31]$ ( $\mathrm{T}_{\mathrm{N}}$ antigen) linked to $\beta^{3}$-homo-serine are known. Despite their importance as specific tumour antigens, conjugates of Fmoc- $\beta^{3}$ hSer and Fmoc- $\beta^{3}$ hThr carrying larger TACA structures such as the Thomsen-Friedenreich antigen (TF) or its sialylated variants $(\alpha 2-6 \mathrm{sTF}$ and $\alpha 2-3 \mathrm{sTF})$ have not been reported to date.

By presenting orthogonally protected $\mathrm{T}_{\mathrm{N}}$ and $\mathrm{TF}$ antigen conjugates of Fmoc- $\beta^{3}$ hThr (Figure 1) as well as a first $\alpha / \beta$ hybrid glycopeptide analogue comprising the 20 amino acid tandem repeat sequence of the human mucin MUC1, we describe preliminary results of our synthetic efforts towards the preparation of mucin-type glycopeptide mimetics.

\section{Results and Discussion}

Initial attempts to directly link the carbohydrate entity to the $\beta$-side chain of a preformed Fmoc- $\beta^{3}$ hThr conjugate were unsuccessful due to rapid lactonisation [29]. Similarly, despite the use of various glycosyl donors and reaction conditions, glycosylation of the corresponding dipeptide precursor Fmoc$\beta^{3}$ hThr(OH)-Ala-OBn failed completely. Therefore we en-

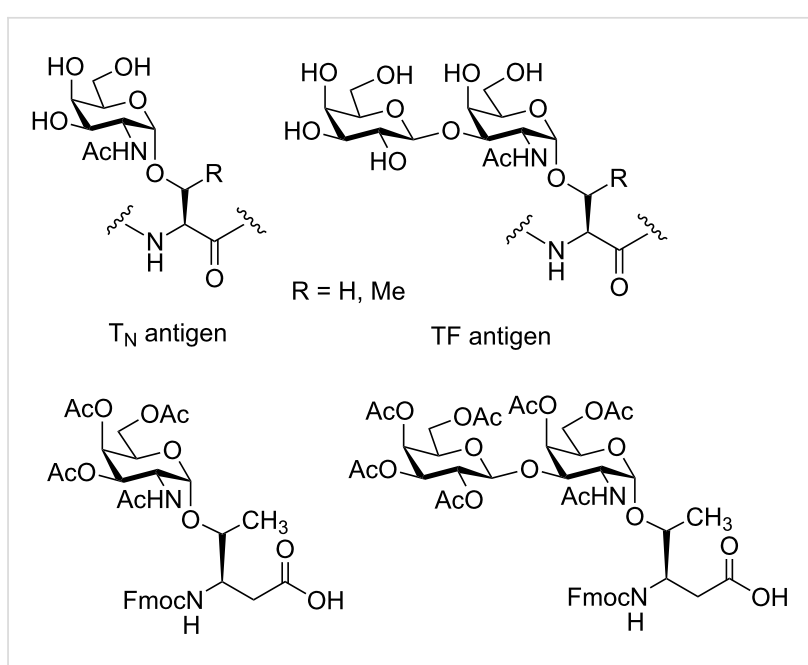

Figure 1: Structures of the naturally occurring $T_{N}$ and TF antigens and the targeted Fmoc- $\beta^{3}$ hThr analogues.

countered the strategy of Arndt-Eistert homologation in the synthesis of the target Fmoc- $\beta^{3}$ hThr $\left(\alpha \mathrm{Ac}_{3}\right.$ GalNAc) and Fmoc$\beta^{3} \mathrm{hThr}\left(\beta\left(\mathrm{Ac}_{4} \mathrm{Gal}(1-3)\right) \alpha\left(\mathrm{Ac}_{3} \mathrm{GalNAc}\right)\right)$ conjugates $\mathbf{2 a}$ and $\mathbf{4}$, respectively, as reported by Norgren et al. [29]. $\mathrm{T}_{\mathrm{N}}$ antigen derivative Fmoc-Thr( $\left.\alpha \mathrm{Ac}_{3} \mathrm{GalNAc}\right)-\mathrm{OH}$ (1a) was prepared according to published procedures $[32,33]$ and converted into the corresponding diazo ketone upon treatment with isobutyl chloroformate in the presence of $N$-methylmorpholine (NMM) and diazomethane (Scheme 1). Without further purification, the latter was subjected to a silver-promoted Wolff-rearrangement, again using NMM as the base, providing the spectroscopically pure $\mathrm{T}_{\mathrm{N}}$ antigen analogue Fmoc- $\beta^{3} \mathrm{hThr}\left(\alpha \mathrm{Ac}_{3} \mathrm{GalNAc}\right)-\mathrm{OH}$ (2a) in $60 \%$ yield over two steps after aqueous work-up.

Compound 2a was also accessible from a direct synthetic precursor of $\mathrm{T}_{\mathrm{N}}$ derivative $\mathbf{1 a}$ in which the 2-acetamido substituent was masked by an azido group. Thus, upon subjection of Fmoc- Thr $\left(\alpha \mathrm{Ac}_{3} \mathrm{GalN}_{3}\right)-\mathrm{OH}$ (1b) [32] to the same homologation sequence as before, the corresponding $\beta^{3} \mathrm{hThr}$ analogue $\mathbf{2 b}$ was obtained in $82 \%$ yield. Subsequent zinc-mediated reduction and acetylation led to the formation of conjugate 2a in $56 \%$ yield.

During biosynthesis $\mathrm{T}_{\mathrm{N}}$ antigen acts as an immediate precursor of the TF antigen. As a consequence, a biomimetic approach towards larger TACA structures via stepwise assembly of the glycan chain has been pursued in various antigen syntheses [33,34]. By applying chemical or enzymatic $3 \beta$-galactosylation, the $3-\mathrm{OH}$ deprotected conjugate $\mathbf{2} \mathbf{b}$ could be converted into the desired antigen derivative Fmoc$\beta^{3} \mathrm{hThr}\left(\beta\left(\mathrm{Ac}_{4} \mathrm{Gal}(1-3)\right) \alpha\left(\mathrm{Ac}_{3} \mathrm{GalNAc}\right)\right)-\mathrm{OH}(4)$. While this strategy certainly requires the use of optimised protecting group manipulations and glycosylation protocols, the alternative route 


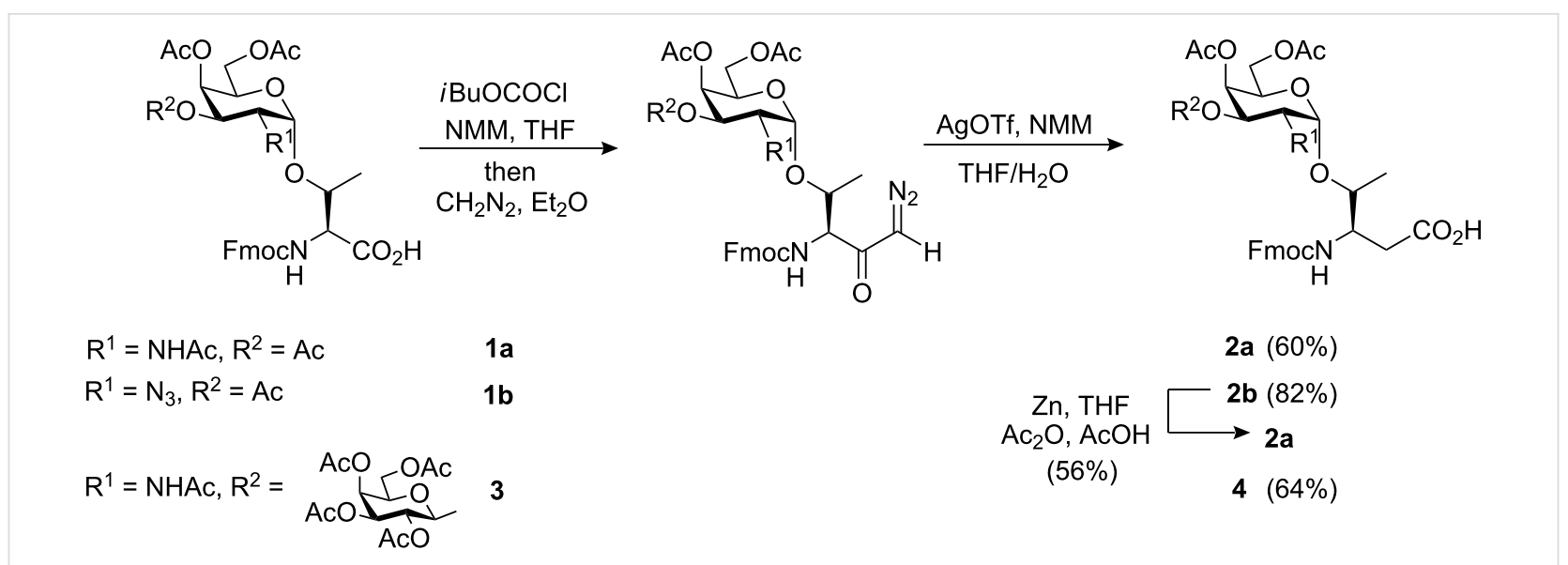

Scheme 1: Synthesis of Fmoc- $\beta^{3}$ hThr antigen conjugates by Arndt-Eistert homologation.

to compound 4 via Arndt-Eistert homologation would benefit from an established and reliable synthesis of key building block 3 [32,33]. To our delight, the homologation reaction of glycosyl amino acid $\mathbf{3}$ again proceeded smoothly to afford the desired TF- $\beta^{3}$ hThr conjugate 4 in spectroscopically pure form and good chemical yield after aqueous work-up (Scheme 1).

To demonstrate the usefulness of the novel glycosylated $\beta^{3} \mathrm{hThr}$ conjugates as antigen mimics, $\mathrm{T}_{\mathrm{N}}$ antigen analog $\mathbf{2 a}$ was incorporated into an $\alpha / \beta$-hybrid glycopeptide 7 comprising a full tandem repeat sequence of the epithelial mucin MUC1 and an $N$-terminal non-immunogenic triethylene glycol spacer. The latter can be used for further conjugation to immunostimulants (e.g., BSA [35] or tetanus toxoid [36]) and for immobilisation onto microarray platforms [37] within functional immunological studies. The MUC1 pseudo-glycopeptide was assembled in an automated synthesiser by the Fmoc-strategy on a TentaGel $\mathrm{S}$ resin 5 equipped with a bulky trityl linker [38] to avoid diketopiperazine formation and pre-loaded with Fmoc-proline (Scheme 2). The first 13 amino acids of the MUC1 sequence were coupled under standard conditions using piperidine in $\mathrm{N}$-methylpyrrolidone (NMP) to remove the temporary Fmoc protecting group followed by coupling of excess (10 equiv) Fmoc-amino acid activated by HBTU/HOBt [39] and diisopropylethylamine (DIPEA) in DMF. Unreacted amino acids were capped after each cycle with $\mathrm{Ac}_{2} \mathrm{O}$ in the presence of DIPEA and catalytic amounts of HOBt in NMP. The sterically demanding glycosylated $\beta^{3}$ hthreonine building block 2a (1.5 equiv), was coupled over an extended reaction time of $8 \mathrm{~h}$ employing the more reactive reagents HATU/HOAt [40] with $N$-methylmorpholine (NMM) in NMP for activation. After the final five Fmoc-amino acids of the TR-sequence were coupled according to the standard protocol, a triethylene glycol spacer 6 [41] (10 equiv) was attached using the standard coupling procedure, again. Simultaneous detachment of the glycopeptide from the resin and cleavage of the acid-labile amino acid side chain protective groups was achieved upon treatment with a mixture of TFA, triisopropylsilane and water. The resulting partially deblocked glycopeptide 7 was isolated after purification by semi-preparative RP-HPLC in a yield of $36 \%$, based on the loaded resin 5 . The final de- $O$-acetylation of the glycan portion was accomplished upon prolonged treatment with catalytic amounts of $\mathrm{NaOMe}$ in methanol at $\mathrm{pH} 9.5$ to afford glycopeptide $\mathbf{8}$ in $18 \%$ yield (based on the loaded resin) after semi-preparative RP-HPLC.

\section{Conclusion}

Two novel tumour-associated carbohydrate antigen analogues with $\mathrm{T}_{\mathrm{N}}$ and $\mathrm{TF}$ determinants $O$-glycosidically linked to the side chain of Fmoc- $\beta^{3}$ hThr-OH have been prepared by Arndt-Eistert homologation of the corresponding glycosylated $\alpha$-amino acids 1a and $\mathbf{1 b}$. The resulting $\beta^{3} \mathrm{hThr}$ glycoconjugates are valuable antigen mimetics with potentially enhanced chemical and metabolic stability. They might serve as precursors for the preparation of further mucin-type antigen structures, e.g., sialylated ones or those based on core 2 structures. In addition, the preparation of a first MUC1 pseudo-glycopeptide comprising the modified glycosyl amino acid Fmoc- $\beta^{3}$ hThr( $\alpha$ GalNAc)-OH at position Thr15 has been accomplished using solid-phase peptide synthesis. By appropriate conjugation, the resulting $\alpha / \beta$ hybrid glycopeptide conjugate could be used as an antigen surrogate to elucidate the effects of chemically modified antibody determinants on the immunological properties of glycopeptide antigen analogues.

\section{Experimental}

General remarks: DMF (amine-free, for peptide synthesis) and NMP were purchased from Roth and $\mathrm{Ac}_{2} \mathrm{O}$ in p.a. quality from Acros. Fmoc-protected amino acids were purchased from 


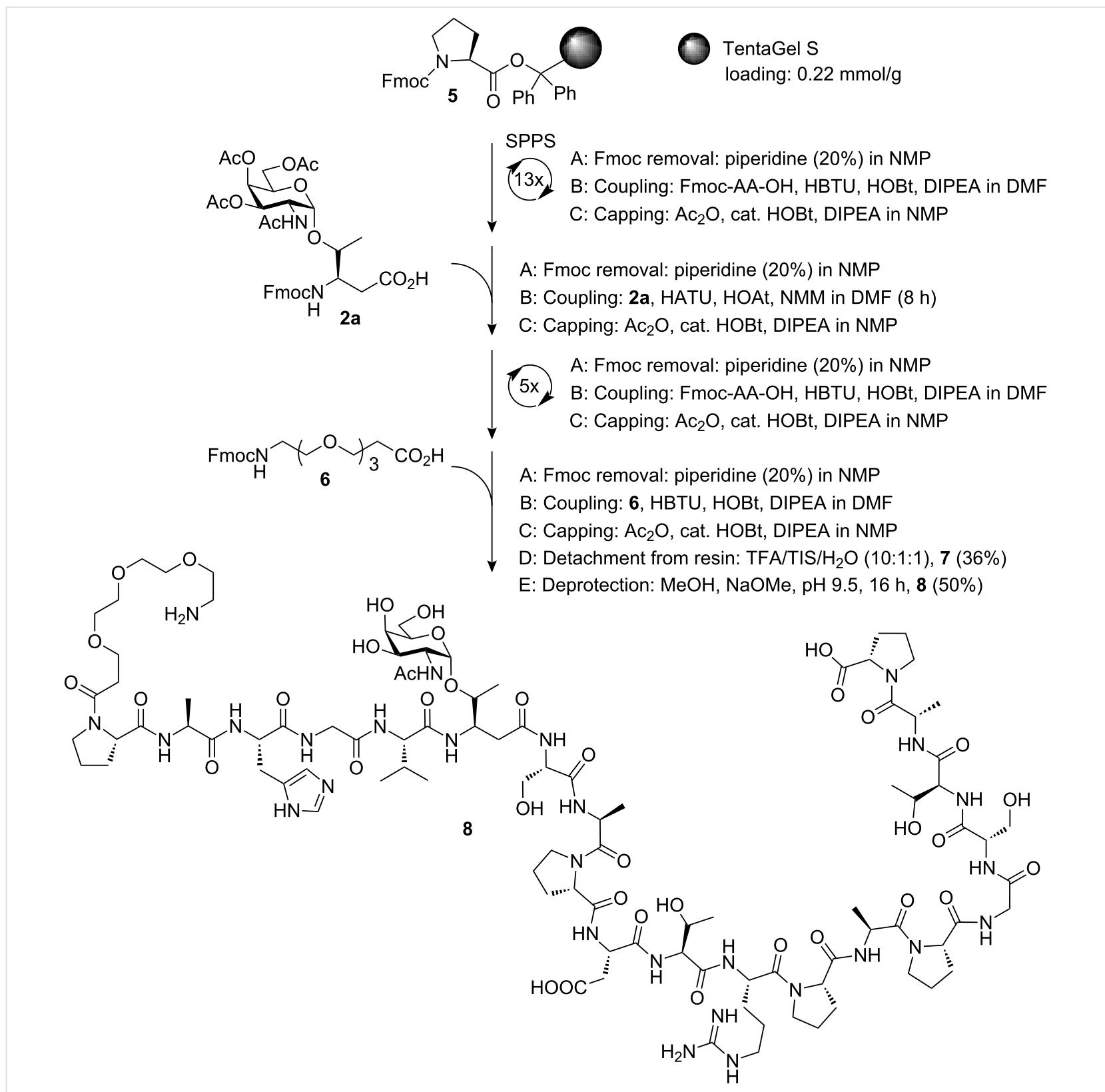

Scheme 2: Solid-phase synthesis of the tumour-associated MUC1 $\alpha / \beta$-hybrid glycopeptide analogue 8 carrying the $T_{N}$ antigen glycan. NMP = $N$-methylpyrrolidone, HBTU $=O-(1 H$-benzotriazol-1-yl $)-N, N, N^{\prime}, N^{\prime}$-tetramethyluronium hexafluorophosphate, HOBt $=N$-hydroxybenzotriazole, DIPEA = diisopropylethylamine, HATU = O-(7-azabenzotriazole-1-yl)- $N, N, N^{\prime}, N^{\prime}$-tetramethyluronium hexafluorophosphate, HOAt = N-hydroxy-7-azabenzotriazole, $\mathrm{NMM}=\mathrm{N}$-methylmorpholine, $\mathrm{TIS}=$ triisopropylsilane.

Orpegen Pharma. For solid-phase synthesis, pre-loaded TentaGel S resin (Rapp Polymere) was employed. Reactions were monitored by TLC with pre-coated silica gel $60 \mathrm{~F}_{254}$ aluminium plates (Merck KGaA, Darmstadt). HPLC analyses were performed on a JASCO-HPLC system with Phenomenex Luna C18(2) $(250 \times 4.6 \mathrm{~mm}, 10 \mu \mathrm{m})$ and Phenomenex Jupiter C18(2) $(250 \times 4.6 \mathrm{~mm}, 10 \mu \mathrm{m})$ columns at a flow rate of $1 \mathrm{~mL}$ $\mathrm{min}^{-1}$. Preparative RP-HPLC separation was carried out on a JASCO-HPLC System with Phenomenex Luna C18(2) (250 × $30 \mathrm{~mm}, 10 \mu \mathrm{m})$ and Phenomenex Jupiter C18(2) $(250 \times 30 \mathrm{~mm}$,
$10 \mu \mathrm{m}$ ) columns at a flow rate of $20 \mathrm{~mL} \mathrm{~min}^{-1}$ or $10 \mathrm{~mL} \mathrm{~min}^{-1}$. ${ }^{1} \mathrm{H},{ }^{13} \mathrm{C}$ and $2 \mathrm{D}$ NMR spectra were recorded on a Bruker AC-300 or a Bruker AM-400 spectrometer. The chemical shifts are reported in ppm relative to the signal of the deuterated solvent. Multiplicities are given as: s (singlet), br s (broad singlet), $d$ (doublet), $t$ (triplet) and $m$ (multiplet). In the case of known compounds, all spectra obtained were consistent with the literature. HR-ESI-mass spectra were recorded on a Micromass Q TOF Ultima 3 spectrometer and optical rotations were measured at $546 \mathrm{~nm}$ with a Perkin-Elmer polarimeter 241 . 


\section{General procedure for the synthesis of diazo- methane in ethereal solution}

Caution: Diazomethane is toxic, highly-volatile, cancerogenic and explosive. Its generation and handling thus requires special precautions [42]. With regard to the free acids, 10 equiv of $\mathrm{N}$-methyl- $\mathrm{N}$-nitroso-urea were added to a solution of $50 \% \mathrm{KOH}$ in $\mathrm{H}_{2} \mathrm{O}$ which was layered on top with $\mathrm{Et}_{2} \mathrm{O}$. The organic layer was decanted and replaced by a new portion of $\mathrm{Et}_{2} \mathrm{O}$ until the organic layer was no longer coloured. The united organic phases were dried over $\mathrm{KOH}$ at $-25^{\circ} \mathrm{C}$ for $3 \mathrm{~h}$.

General procedure (GP1) for the synthesis of diazo ketones: The free acid ( 1 equiv) was dissolved in $2 \mathrm{~mL}$ of dry THF under an argon atmosphere. At $-25^{\circ} \mathrm{C}, 1$ equiv of NMM and 1 equiv of isobutyl chloro formate were added subsequently and the resulting suspension was stirred for $20 \mathrm{~min}$ at this temperature. The mixture was allowed to reach $0{ }^{\circ} \mathrm{C}$ and the diazomethane solution in $\mathrm{Et}_{2} \mathrm{O}$ was added. The yellow solution was stirred 20 min at $0{ }^{\circ} \mathrm{C}$ before it was allowed to reach room temperature and stirred for further $16 \mathrm{~h}$. Excess of diazomethane was destroyed by adding a few drops of acetic acid to the solution until no further nitrogen formation was observed. The solvents were removed under reduced pressure and the residue was dissolved in $20 \mathrm{~mL} \mathrm{Et}_{2} \mathrm{O}$. The organic layer was washed twice with saturated aqueous $\mathrm{NaHCO}_{3}$, saturated aqueous $\mathrm{NH}_{4} \mathrm{Cl}$ and brine. The organic layer was dried over $\mathrm{Na}_{2} \mathrm{SO}_{4}$, filtered, and the solvents were removed under reduced pressure. The resulting diazo ketones were used without further purification.

General procedure (GP2) for the Wolff-rearrangement: The diazo ketone ( 1 equiv) was dissolved in a mixture of $\mathrm{THF} / \mathrm{H}_{2} \mathrm{O}$ (9:1) and was cooled to $0{ }^{\circ} \mathrm{C}$. A solution of 0.11 equiv silver trifluoroacetate in 2.3 equiv NMM was added, and the mixture was stirred for $16 \mathrm{~h}$ while it was allowed to warm to room temperature. After evaporation of THF, the aqueous layer was diluted with saturated aqueous $\mathrm{NaHCO}_{3}$ and $\mathrm{Et}_{2} \mathrm{O}$ was added. The organic layer was extracted three times with saturated aqueous $\mathrm{NaHCO}_{3}$. The aqueous phases were collected, cooled to $0{ }^{\circ} \mathrm{C}$ and acidified to $\mathrm{pH} 1$. The resulting suspension was extracted five times with $\mathrm{Et}_{2} \mathrm{O}$. The organic layer was dried over $\mathrm{Na}_{2} \mathrm{SO}_{4}$ and evaporated in vacuo.

$\mathrm{N}$-(9H-fluoren-9-yl)methoxycarbonyl-[ $\alpha-3,4,6$-tri- $O$-acetyl2-acetamido-2-deoxy-galactopyranosyl]- $\beta^{3}$-homo-threonine 2a: Procedure A: The synthesis followed the general procedures GP1 and GP2. Amounts: $150 \mathrm{mg}(0.22 \mathrm{mmol}) \mathbf{1 a}$. Yield: $90 \mathrm{mg}$ (0.13 mmol), 60\%, colourless amorphous solid. Analytical RP-HPLC (Luna, MeCN- $\mathrm{H}_{2} \mathrm{O}+0.1 \%$ TFA, 20:80 $\left.\rightarrow 60: 40,40 \mathrm{~min}, t_{\mathrm{R}}=29.8 \mathrm{~min}\right)$. Procedure $\boldsymbol{B}:$ To a stirred solution of $\mathbf{2 b}(860 \mathrm{mg}, 0.48 \mathrm{mmol})$ in a mixture of $\mathrm{THF}-\mathrm{Ac}_{2} \mathrm{O}-\mathrm{AcOH}(3: 2: 1,36 \mathrm{~mL})$ activated zinc dust $(0.6 \mathrm{~g}$,
$9.1 \mathrm{mmol}$ ) was added. Activation was achieved by suspension in aq. $2 \%$ solution of $\mathrm{CuSO}_{4}$, followed by subsequent washings with water, EtOAc and $\mathrm{Et}_{2} \mathrm{O}$. The reaction mixture was stirred for $16 \mathrm{~h}$ at room temperature, diluted with $50 \mathrm{~mL}$ THF and filtered through Hyflo Supercel ${ }^{\circledR}$. The filtrate was concentrated in vacuo and co-evaporated five times with toluene and $\mathrm{CH}_{2} \mathrm{Cl}_{2}$. The residue was dissolved in $50 \mathrm{~mL} \mathrm{CH}_{2} \mathrm{Cl}_{2}$, washed with saturated aqueous $\mathrm{NaHCO}_{3}$ and brine, dried $\left(\mathrm{Na}_{2} \mathrm{SO}_{4}\right)$ and concentrated in vacuo. Yield: $328 \mathrm{mg}(0.48 \mathrm{mmol}), 56 \%$, colourless amorphous solid; $[\alpha]_{\mathrm{D}}{ }^{23}=42.6\left(c=1.00, \mathrm{CHCl}_{3}\right)$; ${ }^{1} \mathrm{H}$ NMR (COSY, HSQC, $400 \mathrm{MHz}, \mathrm{CD}_{3} \mathrm{OD}$ ), $\delta(\mathrm{ppm})=7.81$ (d, 2 H, $J=7.2$ Hz, Fmoc-H4, Fmoc-H5), 7.66, 7.65 (2d, 2 H, $J=7.6 \mathrm{~Hz}$, Fmoc-H1, Fmoc-H8), 7.39 (pt, 2 H, Fmoc-H3, Fmoc-H6), 7.32 (t, $2 \mathrm{H}, J=7.6 \mathrm{~Hz}$, Fmoc-H2, Fmoc-H7), 5.44 (d, $1 \mathrm{H}, J=2.4 \mathrm{~Hz}$, GalNAc-H4), $5.10(\mathrm{dd}, 1 \mathrm{H}, J=3.2 \mathrm{~Hz}$, $11.6 \mathrm{~Hz}$, GalNAc-H3), 4.98 (d, $1 \mathrm{H}, J=3.6 \mathrm{~Hz}$, GalNAc-H1), 4.56 (pdd, $1 \mathrm{H}$, Fmoc-CH $), 4.45$ (dd, $1 \mathrm{H}, J=3.6 \mathrm{~Hz}, 11.6 \mathrm{~Hz}$, GalNAc-H2), 4.41-4.35 (m, $1 \mathrm{H}$, Fmoc- $\left.\mathrm{CH}_{2}\right), 4.31$ (pt, $1 \mathrm{H}$, GalNAc-H5), 4.21 (pt, 1 H, Fmoc-H9), 4.15-4.07 (m, 2 H, GalNAc-H6), 4.05-4.02 (m, 1 H, hT $\left.{ }^{\beta}\right), 3.83$ (pdd, $1 \mathrm{H}, \mathrm{hT}^{\gamma}$ ), $2.55\left(\mathrm{pdd}, 1 \mathrm{H}, \mathrm{hT}^{\alpha}\right), 2.38\left(\mathrm{pdd}, 1 \mathrm{H}, \mathrm{hT}^{\alpha}\right), 1.76(\mathrm{~d}, 3 \mathrm{H}$, $\left.J=6.4 \mathrm{~Hz}, \mathrm{hT}^{\delta}\right) ;{ }^{13} \mathrm{C}$ NMR (DEPT, HSQC, $100.6 \mathrm{MHz}$, $\left.\mathrm{CD}_{3} \mathrm{OD}\right), \delta(\mathrm{ppm})=175.1,172.2,170.7,170.7,170.6(\mathrm{C}=\mathrm{O})$, 157.0 (C=O-urethane), 144.0, 143.8 (Fmoc-C1a, Fmoc-C8a), 141.3, 141.3 (Fmoc-C4a, Fmoc-C5a), 127.4 (Fmoc-C3, FmocC6), 126.8, 126.7 (Fmoc-C2, Fmoc-C7), 124.7, 124.6 (FmocC1, Fmoc-C8), 119.6, 119.5 (Fmoc-C4, Fmoc-C5), 99.1 (GalNAc-C1), $77.6\left(\mathrm{hT}^{\gamma}\right), 68.3$ (GalNAc-C3), 67.4 (GalNAcC4), 66.6 (GalNAc-C5), 65.9 (Fmoc-CH ${ }_{2}$ ), 61.8 (GalNAc-C6), $52.6\left(\mathrm{hT}^{\beta}\right), 47.6$ (GalNAc-C2), 47.3 (Fmoc-C9), $36.8\left(\mathrm{hT}^{\alpha}\right)$, $21.3\left(\mathrm{CH}_{3}-\mathrm{NHAc}\right), 19.3,19.2,19.1\left(\mathrm{CH}_{3}-\mathrm{Ac}\right), 16.7\left(\mathrm{hT}^{\delta}\right)$; HR-ESI-MS (positive, $\mathrm{m} / \mathrm{z}): 707.2433\left([\mathrm{M}+\mathrm{Na}]^{+}\right.$, calc.: 707.2428).

$\mathrm{N}$-(9H-fluoren-9-yl)methoxycarbonyl-[ $\alpha-3,4,6$-tri- $\mathrm{O}$-acetyl2-azido-2-deoxy-galactopyranosyl]- $\boldsymbol{\beta}^{3}$-homo-threonine $\mathbf{2 b}$ : The synthesis followed the general procedures GP1 and GP2. Amounts: $156 \mathrm{mg}$ (0.23 mmol) 1b. Yield: $130 \mathrm{mg}(0.19 \mathrm{mmol})$, $82 \%$, colourless amorphous solid; $[\alpha]_{\mathrm{D}}{ }^{23}=56.0(c=1.00$, $\mathrm{CHCl}_{3}$ ); ${ }^{1} \mathrm{H} \mathrm{NMR}\left(400 \mathrm{MHz}, \mathrm{COSY}, \mathrm{HSQC}, \mathrm{CD}_{3} \mathrm{OD}\right), \delta(\mathrm{ppm})$ $=7.79(\mathrm{~d}, 2 \mathrm{H}, J=7.6 \mathrm{~Hz}$, Fmoc-H4, Fmoc-H5), $7.66(\mathrm{~d}, 2 \mathrm{H}$, $J=7.6 \mathrm{~Hz}$, Fmoc-H1, Fmoc-H8), 7.39 (t, $2 \mathrm{H}, J=7.2 \mathrm{~Hz}$, Fmoc-H3, Fmoc-H6), 7.32 (t, $2 \mathrm{H}, J=7.2 \mathrm{~Hz}$, Fmoc-H2, FmocH7), 5.44 (d, $1 \mathrm{H}, J=2.0 \mathrm{~Hz}$, GalNAc-H4), 5.33 (dd, $1 \mathrm{H}, J=$ $3.2 \mathrm{~Hz}, 11.2 \mathrm{~Hz}$, GalNAc-H3), 5.14 (d, $1 \mathrm{H}, J=3.6 \mathrm{~Hz}$, GalNAc-H1), 4.41-4.32 (m, 3 H, Fmoc- $\mathrm{CH}_{2}$, Fmoc-H9), 4.21 (pt, $1 \mathrm{H}$, GalNAc-H5), 4.14-4.06 (m, $3 \mathrm{H}$, GalNAc-H6, hT ${ }^{\beta}$ ), 3.91-3.86 (m, $2 \mathrm{H}$, GalNAc-H2, hT $\left.{ }^{\gamma}\right), 2.76(\mathrm{dd}, 1 \mathrm{H}, J=5.2 \mathrm{~Hz}$, $\left.16 \mathrm{~Hz}, \mathrm{hT}^{\alpha}\right), 2.55\left(\mathrm{dd}, 1 \mathrm{H}, J=8.8 \mathrm{~Hz}, 16.4 \mathrm{~Hz}, \mathrm{hT}^{\alpha}\right), 2.14$, 2.02, $1.98\left(3 \mathrm{~s}, 9 \mathrm{H}, 3 \times \mathrm{CH}_{3}-\mathrm{Ac}\right) ; 1.76\left(\mathrm{~d}, 3 \mathrm{H}, J=6.4 \mathrm{~Hz}, \mathrm{hT}^{\delta}\right)$; ${ }^{13} \mathrm{C}$ NMR (101 MHz, DEPT, HSQC, $\left.\mathrm{CD}_{3} \mathrm{OD}\right), \delta(\mathrm{ppm})=$ 
173.5, 170.7, 170.6, 170.1 (C=O), $157.1(\mathrm{C}=\mathrm{O}$-urethane), 144.0, 143.9 (Fmoc-C1a, Fmoc-C8a), 141.2 (Fmoc-C4a, Fmoc-C5a), 127.4 (Fmoc-C3, Fmoc-C6), 126.8, 126.7 (Fmoc-C2, FmocC7), 124.9 (Fmoc-C1, Fmoc-C8), 119.5 (Fmoc-C4, Fmoc-C5), 98.6 (GalNAc-C1), $77.5\left(\mathrm{hT}^{\gamma}\right), 69.0$ (GalNAc-C3), 67.7 (GalNAc-C4), 66.8 (GalNAc-C5), 66.4 (Fmoc-CH 2$), 58.4$ (GalNAc-C6), 52.0 (hT ${ }^{\gamma}$ ), 47.6 (GalNAc-C2), 47.3 (Fmoc-C9), $34.8\left(\mathrm{hT}^{\alpha}\right), 19.3,19.2,19.1\left(\mathrm{CH}_{3}-\mathrm{Ac}\right), 16.4\left(\mathrm{hT}^{\delta}\right)$; HR-ESI-MS (positive, $\mathrm{m} / \mathrm{z}): 691.2232\left([\mathrm{M}+\mathrm{Na}]^{+}\right.$calc.: 691.2227).

$\mathrm{N}$-(9H-fluoren-9-yl)methoxycarbonyl-[( $\beta-2,3,4,6$-tetra- $O$ acetylgalactopyranosyl)-(1-3)-( $\alpha$-2-acetamido-4,6-di- $O$ acetyl-2-deoxy-galactopyranosyl)]- $\beta^{3}$-homo-threonine 4 : The synthesis followed the general procedures GP1 and GP2. Amounts: $200 \mathrm{mg}(0.21 \mathrm{mmol})$ 3. Yield: $130.3 \mathrm{mg}(0.13 \mathrm{mmol})$, $64 \%$, colourless amorphous solid; $[\alpha]_{\mathrm{D}}{ }^{23}=29.9(c=0.50$, $\mathrm{CHCl}_{3}$ ); ${ }^{1} \mathrm{H} \mathrm{NMR}\left(400 \mathrm{MHz}, \mathrm{COSY}, \mathrm{HSQC}, \mathrm{CD}_{3} \mathrm{OD}\right), \delta(\mathrm{ppm})$ $=7.85(\mathrm{~d}, 2 \mathrm{H}, J=7.6 \mathrm{~Hz}$, Fmoc-H4, Fmoc-H5), 7.69 (d, $2 \mathrm{H}$, $J=7.6 \mathrm{~Hz}$, Fmoc-H1, Fmoc-H8), 7.44 (t, $2 \mathrm{H}, J=7.6 \mathrm{~Hz}$, Fmoc-H3, Fmoc-H6), 7.36 (t, $2 \mathrm{H}, J=7.6 \mathrm{~Hz}$, Fmoc-H2, FmocH7), 5.43 (d, $1 \mathrm{H}, J=3.4 \mathrm{~Hz}$, GalNAc-H4), 5.39 (m, $1 \mathrm{H}, \mathrm{Gal}-$ H3), 5.04-5.02 (m, 2 H, Gal-H4, Gal-H2), 4.98 (under $\mathrm{H}_{2} \mathrm{O}-$ peak, GalNAc-H1), 4.71 (m, 1H, Gal-H1), 4.51 (d, $2 \mathrm{H}, J=6.2$ Hz, Gal-H6), 4.44 (dd, $1 \mathrm{H}, J=3.5 \mathrm{~Hz}, 11.5 \mathrm{~Hz}$, GalNAc-H2), 4.28-4.23 (m, 2 H, Gal-H5, Fmoc-H9), 4.23-4.21 (m, 3 H, Fmoc- $\mathrm{CH}_{2}$, GalNAc-H6), 4.09-3.91 (m, 4 H, GalNAc-H5, Fmoc- $\mathrm{CH}_{2}, \mathrm{hT}^{\beta}$, GalNAc-H3), 3.86 (dd, $1 \mathrm{H}, J=2.7 \mathrm{~Hz}, 6.2$ $\left.\mathrm{Hz}, \mathrm{hT}^{\gamma}\right), 2.71\left(\mathrm{dd}, 1 \mathrm{H}, J=6.3 \mathrm{~Hz}, 15.5 \mathrm{~Hz}, \mathrm{hT}^{\alpha}\right), 2.55(\mathrm{dd}, 1$ $\left.\mathrm{H}, J=8.7 \mathrm{~Hz}, 15.6 \mathrm{~Hz}, \mathrm{hT}^{\alpha}\right), 2.17,2.15,2.06,2.06,2.05,2.04$, $1.97\left(7 \mathrm{~s}, 21 \mathrm{H}, 6 \times \mathrm{CH}_{3}-\mathrm{Ac}, \mathrm{CH}_{3}-\mathrm{AcNH}\right), 1.22(\mathrm{~d}, 3 \mathrm{H}, J=6.6$ $\left.\mathrm{Hz}, \mathrm{hT}^{\delta}\right) ;{ }^{13} \mathrm{C}$ NMR (101 MHz, DEPT, HSQC, $\left.\mathrm{CD}_{3} \mathrm{OD}\right), \delta$ $(\mathrm{ppm})=174.7,173.0,172.5,172.2,171.6,171.2(\mathrm{C}=\mathrm{O}), 158.5$ $(\mathrm{C}=\mathrm{O}$-urethane), 145.5, 145.3 (Fmoc-C1a, Fmoc-C8a), 142.8, 142.8 (Fmoc-C4a, Fmoc-C5a), 129.0, 128.4 (Fmoc-C3, FmocC6), 126.3, 126.2 (Fmoc-C2, Fmoc-C7), 121.2 (Fmoc-C1, Fmoc-C8, Fmoc-C4, Fmoc-C5), 102.6 (Gal-C1), 100.6 (GalNAc-C1), $78.2\left(\mathrm{hT}^{\gamma}\right), 74.9$ (GalNAc-C3), 72.2 (Gal-C4), 71.8 (GalNAc-C5), 71.2 (GalNAc-C4), 70.1 (Gal-C2), 68.8 (Gal-C5), 68.5 (Gal-C3), 67.4 (Gal-C6), 64.3 (Fmoc-CH 2 ), 62.3 (GalNAc-C6), $53.6\left(\mathrm{hT}^{\beta}\right.$ ), 50.3 (GalNAc-C2), 36.5 (hT $^{\alpha}$ ), 23.0 ( $\mathrm{CH}_{3}$-NHAc), 20.8, 20.8, 20.7, $20.5\left(\mathrm{CH}_{3}-\mathrm{Ac}\right), 18.0\left(\mathrm{hT}^{\delta}\right)$; HR-ESI-MS (positive, $\mathrm{m} / \mathrm{z})$ : $1159.3216\left([\mathrm{M}+\mathrm{Na}]^{+}\right.$, calc.: 1159.3210).

Amino-4,7,10-trioxadodecanylamido- $N$-L-prolyl-L-alanylL-histidyl-L-glycyl-L-valyl- $O$-[ $\alpha-3,4,6$-tri- $O$-acetyl-2-acetamido-2-deoxy-galactopyranosyl]-homo- $\beta^{3}$-threonyl-Lseryl-L-alanyl-L-prolyl-L-aspartyl-L-threonyl-L-arginyl-Lprolyl-L-alanyl-L-prolyl-L-glycyl-L-seryl-L-threonyl-Lalanyl-L-proline 7: The synthesis was carried out in an Applied Biosystems ABI 433A peptide synthesiser (standard program Fastmoc $0.1 \mathrm{mmol}$ ) using pre-loaded Fmoc-Pro-TrtTentaGel S resin (455 mg, $0.10 \mathrm{mmol}$; loading: $0.22 \mathrm{mmol} / \mathrm{g}$ ). For the coupling reactions, the amino acids Fmoc-Ala-OH, Fmoc-Arg(Pmc)-OH, Fmoc-Asp-OH, Fmoc-Gly-OH, FmocHis(Trt)-OH, Fmoc-Pro-OH, Fmoc-Ser( $t \mathrm{Bu})-\mathrm{OH}$, Fmoc$\operatorname{Thr}(t \mathrm{Bu})-\mathrm{OH}$, and Fmoc-Val-OH were employed. In every coupling cycle, the $\mathrm{N}$-terminal Fmoc group was removed by treatment of the resin with a solution of piperidine $(20 \%)$ in NMP for at least $3 \times 2.5 \mathrm{~min}$. The coupling of the amino acids ( $1 \mathrm{mmol}$ or 10 equiv based on the loaded resin) was carried out with HBTU (1 mmol), HOBt ( $1 \mathrm{mmol})$ and DIPEA (2 mmol) in DMF (20-30 min vortex). After every coupling step, unreacted amino groups were capped by treatment with a mixture of $\mathrm{Ac}_{2} \mathrm{O}$ $(0.5 \mathrm{M})$, DIPEA $(0.125 \mathrm{M})$ and HOBt $(0.015 \mathrm{M})$ in NMP (10 min vortex). Coupling of the glycosylated $\beta^{3} \mathrm{hThr}$ building block $2 \mathrm{a}$ (102.70 mg, $0.15 \mathrm{mmol}$ ) was performed using HATU (1.2 equiv with respect to the glycosyl amino acid), HOAt (1.2 equiv) and NMM (4 equiv) for activation ( $8 \mathrm{~h}$ vortex). After coupling of the remaining five amino acids by the standard procedure, the triethylene glycol spacer (1 mmol, 10 equiv based on the loaded resin) was coupled using HBTU ( $1 \mathrm{mmol})$, HOBt $(1 \mathrm{mmol})$ and DIPEA $(2 \mathrm{mmol})$ in DMF (20-30 min vortex) and the $N$-terminal Fmoc group was removed by piperidine (20\%) in NMP. Detachment from the resin and simultaneous removal of all side chain protecting groups was performed in a Merrifield glass reactor by shaking with TFA $(10 \mathrm{~mL})$, TIS $(1.0 \mathrm{~mL})$ and $\mathrm{H}_{2} \mathrm{O}(1.0 \mathrm{~mL})$ for $3 \mathrm{~h}$. The solution was filtered, the resin was washed with $\mathrm{CH}_{2} \mathrm{Cl}_{2}$ $(5 \times 10 \mathrm{~mL})$ and the combined solutions were concentrated in vacuo to a volume of $0.5 \mathrm{~mL}$. After co-evaporation with toluene $(3 \times 10 \mathrm{~mL})$, the crude product was dissolved in $\mathrm{H}_{2} \mathrm{O}$ and subjected to lyophilisation. The crude product was purified by RP-HPLC (Luna, MeCN- $\mathrm{H}_{2} \mathrm{O}+$ 0.1\% TFA, 5:95 $\rightarrow$ 100:0, $\left.50 \mathrm{~min}, t_{\mathrm{R}}=25.2 \mathrm{~min}\right)$. Yield: $88.3 \mathrm{mg}(0.04 \mathrm{mmol}), 36 \%$, colourless amorphous solid; $[\alpha]_{\mathrm{D}}{ }^{23}=-79.9\left(c=1.00, \mathrm{H}_{2} \mathrm{O}\right) ;{ }^{1} \mathrm{H}$ NMR (400 MHz, COSY, HSQC, TOCSY, $\left.\mathrm{D}_{2} \mathrm{O}\right), \delta(\mathrm{ppm}): 8.50$ $\left(\mathrm{m}, 1 \mathrm{H}, \mathrm{H}^{\varepsilon}\right), 7.19\left(\mathrm{~m}, 1 \mathrm{H}, \mathrm{H}^{\delta}\right), 5.31-5.28(\mathrm{~m}, 1 \mathrm{H}$, GalNAcH4), 5.08 (dd, $1 \mathrm{H}, J=3.1 \mathrm{~Hz}, 11.7 \mathrm{~Hz}$, GalNAc-H3), 5.01 (d, 1 $\mathrm{H}, J=3.5 \mathrm{~Hz}$, GalNAc-H1), 4.64-4.54 (m, $2 \mathrm{H}, \mathrm{D}^{\alpha}\{4.61\}, \mathrm{H}^{\alpha}$ $\{4.57\}), 4.54-4.37\left(\mathrm{~m}, 5 \mathrm{H}, \mathrm{R}^{\alpha}\{4.52\}, \mathrm{A}_{2}^{\alpha}\{4.47\}, \mathrm{A}_{3}{ }^{\alpha}\{4.46\}\right.$, $\left.\mathrm{A}_{4}{ }^{\alpha}\{4.45\}, \mathrm{S}_{1}{ }^{\alpha}\{4.39, \mathrm{t}, J=5.5 \mathrm{~Hz}\}\right), 4.36-4.20\left(\mathrm{~m}, 10 \mathrm{H}, \mathrm{S}_{2}{ }^{\alpha}\right.$ $\{4.33\}, \mathrm{hT}^{\beta}\{4.27\}, \mathrm{A}_{1}^{\alpha}\{4.29\}$, GalNAc-H5 $\{4.32\}, \mathrm{T}_{1}^{\alpha}$ $\left.\{4.30\}, \mathrm{T}_{2}{ }^{\alpha}\{4.24\}, \mathrm{P}_{1-4^{\alpha}}\{4.29\}\{4.28\}\{4.27\}\{4.24\}\right), 4.19$ $\left(\mathrm{d}, 1 \mathrm{H}, J=4.5 \mathrm{~Hz}, \mathrm{~V}^{\alpha}\right), 4.15-3.89(\mathrm{~m}, 6 \mathrm{H}$, GalNAc-H2 $\{4.12\}, \mathrm{T}_{1}{ }^{\beta}, \mathrm{T}_{2}{ }^{\beta}\{4.09\}\{4.07\}$, GalNAc-H6 $\{4.02\}, \mathrm{P}_{5}^{\alpha}$ $\{3.94\}), 3.89-3.73\left(\mathrm{~m}, 7 \mathrm{H}, \mathrm{G}_{1}{ }^{\alpha}\{3.86\}, \mathrm{G}_{2}{ }^{\alpha}\{3.83\}, \mathrm{G}_{1}{ }^{\alpha}\right.$ $\left.\{3.80\}, \mathrm{G}_{2}{ }^{\alpha}\{3.77\}, \mathrm{hT}^{\gamma}\{3.77\}, \mathrm{S}_{2}{ }^{\beta}\{3.76\}\right), 3.73-3.59(\mathrm{~m}, 11$ $\mathrm{H}, \mathrm{P}_{1-5} \delta\{3.67\}, \mathrm{S}_{1}{ }^{\beta}\{3.65\}, 2 \times \mathrm{CH}_{2}$-spacer $\left.\{3.64\}\{3.62\}\right)$, 3.59-3.28 (m, $13 \mathrm{H}, 2 \times \mathrm{CH}_{2}$-spacer $\{3.57\}, 2 \times \mathrm{CH}_{2}$-spacer $\left.\{3.54\}, \mathrm{P}_{1-5} \delta\{3.52\}\right), 3.18\left(\mathrm{dd}, 1 \mathrm{H}, J=5.2 \mathrm{~Hz}, 15.1 \mathrm{~Hz}, \mathrm{H}^{\beta}\right)$, 3.11-3.01 (m, $5 \mathrm{H}, \mathrm{CH}_{2}$-spacer $\left.\{3.08\}, \mathrm{H}^{\beta}\{3.07\}, \mathrm{R}^{\delta}\{3.07\}\right)$, 
$2.84\left(\mathrm{dd}, 1 \mathrm{H}, J=6.5 \mathrm{~Hz}, 16.8 \mathrm{~Hz}, \mathrm{D}^{\beta}\right), 2.75(\mathrm{dd}, 1 \mathrm{H}, J=$ $\left.6.5 \mathrm{~Hz}, 17.3 \mathrm{~Hz}, \mathrm{D}^{\beta}\right), 2.67-2.40\left(\mathrm{~m}, 4 \mathrm{H}, \mathrm{CH}_{2}\right.$-spacer $\{2.61\}$ $\left.\{2.55\}, \mathrm{hT}^{\alpha}\{2.59\}\{2.46\}\right), 2.23-2.05\left(\mathrm{~m}, 8 \mathrm{H}, \mathrm{P}_{1-5}{ }^{\beta}\{2.20\}\right.$ $\left.\{2.17\}\{2.14\}, \mathrm{CH}_{3}-\mathrm{Ac}\{2.07\}\right), 2.01-1.66\left(\mathrm{~m}, 29 \mathrm{H}, 4 \times \mathrm{CH}_{3}-\right.$ Ac $\left.\{1.94\}\{1.92\}\{1.89\}\{1.88\}, \mathrm{P}_{1-5}{ }^{\gamma}\{1.95-1.83\}\right), \mathrm{P}_{1-5}{ }^{\beta}$ $\left.\{1.92-1.71\}, \mathrm{V}^{\beta}\{1.86\}, \mathrm{R}^{\beta}\{1.70\}\right), 1.65-1.46\left(\mathrm{~m}, 3 \mathrm{H}, \mathrm{R}^{\beta}\right.$ $\left.\{1.61\}, \mathrm{R}^{\gamma}\{1.54\}\right), 1.28-1.17\left(\mathrm{~m}, 12 \mathrm{H}, \mathrm{A}_{1-4}{ }^{\beta}\{1.24\}\{1.20\}\right)$ 1.07-1.01 (m, $\left.9 \mathrm{H}, \mathrm{T}_{1}^{\gamma}\{1.07\}, \mathrm{hT}^{\delta}\{1.06\}, \mathrm{T}_{2}^{\gamma}\{1.04\}\right), 0.76$ $\left(\mathrm{d}, 3 \mathrm{H}, J=5.8 \mathrm{~Hz}, \mathrm{~V}^{\gamma}\right), 0.71\left(\mathrm{~d}, 3 \mathrm{H}, J=6.4 \mathrm{~Hz}, \mathrm{~V}^{\gamma}\right) ;{ }^{13} \mathrm{C}$ NMR (101 MHz, DEPT, HSQC, $\left.\mathrm{D}_{2} \mathrm{O}\right), \delta(\mathrm{ppm})=176.6,175.7$, 174.9, 174.4, 174.3, 173.9, 173.43, 173.4, 173.1, 172.9, 172.8, 172.6, 172.4, 172.3, 171.9, 171.5, 171.3, 171.2, 171.1, 170.9, 170.8, $170.7(\mathrm{C}=\mathrm{O}), 163.3,163.0,162.6,162.2$ (TFA), 156.7 $(\mathrm{C}=\mathrm{NH}), 133.5\left(\mathrm{H}^{\beta}\right), 128.4\left(\mathrm{H}^{\varepsilon}\right), 117.3\left(\mathrm{H}^{\delta}\right), 98.2,98.0$ (GalNAc-C1), 77.2, $76.9\left(\mathrm{hT}^{\gamma}\right), 69.6,69.5,69.4,69.4\left(\mathrm{CH}_{2-}\right.$ Spacer), 68.4 (GalNAc-C3), 68.0 (GalNAc-C2), $67.0\left(\mathrm{~T}_{1}{ }^{\beta}\right.$, $\left.\mathrm{T}_{2}^{\beta}\right)$, 66.3, 66.0 ( $\mathrm{CH}_{2}$-spacer), 62.5 (GalNAc-C6), 61.5, 61.1 $\left(\mathrm{S}_{1}{ }^{\beta}, \mathrm{S}_{2}{ }^{\beta}\right), 60.8,60.5,60.0,59.7,58.7\left(\mathrm{P}_{1-5}{ }^{\alpha}\right), 59.6,59.3\left(\mathrm{~T}_{1}^{\alpha}\right.$, $\left.\mathrm{T}_{2}{ }^{\alpha}\right), 58.9\left(\mathrm{~V}^{\alpha}\right), 55.5,54.9,52.24\left(\mathrm{H}^{\alpha}\right), 51.1\left(\mathrm{R}^{\alpha}\right), 50.5\left(\mathrm{hT}^{\beta}\right)$, $50.0\left(\mathrm{D}^{\alpha}\right), 49.6$ (GalNAc-C2), 48.0, 47.9, 47.7, $47.4\left(\mathrm{P}_{1-5}{ }^{\delta}\right)$, 47.8, 47.8, 47.6, $47.6\left(\mathrm{~A}_{1}{ }^{\alpha}, \mathrm{A}_{2}{ }^{\alpha}, \mathrm{A}_{3}{ }^{\alpha}, \mathrm{A}_{4}{ }^{\alpha}\right), 42.4,42.3\left(\mathrm{G}_{1}^{\alpha}\right.$, $\left.\mathrm{G}_{2}{ }^{\alpha}\right), 40.5\left(\mathrm{CH}_{2}\right.$-spacer), $39.0\left(\mathrm{R}^{\delta}\right), 36.2\left(\mathrm{hT}^{\alpha}\right), 34.9\left(\mathrm{D}^{\beta}\right), 34.0$ ( $\mathrm{CH}_{2}$-spacer), 29.9, $29.8\left(\mathrm{~V}^{\beta}\right), 29.6,29.3,29.2,29.2,28.1$ $\left(\mathrm{P}_{1-5}{ }^{\beta}\right), 27.4\left(\mathrm{R}^{\beta}\right), 26.2\left(\mathrm{H}^{\beta}\right), 24.7,24.6,24.5,24.3\left(\mathrm{P}_{1-5^{\gamma}}\right), 23.9$ $\left(\mathrm{R}^{\gamma}\right), 21.8\left(\mathrm{CH}_{3}-\mathrm{AcNH}\right), 20.3,20.0,20.0\left(\mathrm{CH}_{3}-\mathrm{Ac}\right), 18.8,18.7$ $\left(\mathrm{T}_{1}^{\gamma}, \mathrm{T}_{2}^{\gamma}\right), 18.3,17.7\left(\mathrm{~V}^{\gamma \alpha} \mathrm{V}^{\gamma \beta}\right), 16.6\left(\mathrm{hT}^{\delta}\right), 16.2,15.4,15.2$ $15.1\left(\mathrm{~A}_{1-4}{ }^{\beta}\right)$; HR-ESI-MS: $1217.0941\left([\mathrm{M}+2 \mathrm{H}]^{+}\right.$, calc.: 1217.0932).

Amino-4,7,10-trioxadodecanylamido- $N$-L-prolyl-L-alanylL-histidyl-L-glycyl-L-valyl- $O$-[ $\alpha$-2-acetamido-2-deoxygalactopyranosyl]-homo- $\beta^{3}$-threonyl-L-seryl-L-alanyl-Lprolyl-L-aspartyl-L-threonyl-L-arginyl-L-prolyl-L-alanylL-prolyl-L-glycyl-L-seryl-L-threonyl-L-alanyl-L-proline 8: Peptide 7 was dissolved in $10 \mathrm{~mL}$ of methanol (HPLC grade). A fresh solution of sodium methanolate in methanol $(0.5 \mathrm{~g} \mathrm{Na}$ in $25 \mathrm{~mL}$ methanol (HPLC grade)) was added drop wise until $\mathrm{pH}$ 9.5 was reached. The reaction mixture was stirred over night and neutralised with a few drops of acetic acid. The solvent was removed in vacuo and the residue was dissolved in $\mathrm{H}_{2} \mathrm{O}$ and subjected to lyophilisation. The crude product was purified by RP-HPLC (Luna, MeCN- $\mathrm{H}_{2} \mathrm{O}+$ 0.1\% TFA, 5:95 $\rightarrow$ 100:0, $\left.60 \mathrm{~min}, t_{\mathrm{R}}=15.3 \mathrm{~min}\right)$. Yield: $44 \mathrm{mg}(0.02 \mathrm{mmol}), 50 \%(18 \%$ based on the loaded resin 5), colourless amorphous solid; $[\alpha]_{\mathrm{D}}{ }^{23}$ $=-83.7\left(c=1.00, \mathrm{H}_{2} \mathrm{O}\right) ;{ }^{1} \mathrm{H} \mathrm{NMR}(400 \mathrm{MHz}, \mathrm{COSY}$, HSQC, $\left.\mathrm{D}_{2} \mathrm{O}\right), \delta(\mathrm{ppm})=8.50\left(\mathrm{~d}, 1 \mathrm{H}, J=1.6 \mathrm{~Hz}, \mathrm{H}^{\delta}\right), 7.20(\mathrm{~d}, 1 \mathrm{H}, J=$ $\left.1.3 \mathrm{~Hz}, \mathrm{H}^{\varepsilon}\right), 4.89(\mathrm{~d}, 1 \mathrm{H}, J=3.9 \mathrm{~Hz}$, GalNAc-H1), $4.62(\mathrm{t}, 1 \mathrm{H}$, $\left.J=6.8 \mathrm{~Hz}, \mathrm{D}^{\alpha}\right), 4.58\left(\mathrm{dd}, 1 \mathrm{H}, J=6.7 \mathrm{~Hz}, 8.6 \mathrm{~Hz}, \mathrm{H}^{\alpha}\right)$, 4.55-4.49(m, $\left.1 \mathrm{H}, \mathrm{R}^{\alpha}\right), 4.50-4.43\left(\mathrm{~m}, 3 \mathrm{H}, \mathrm{A}_{1-3}{ }^{\alpha}\{4.48\}\right.$ $\{4.47\}\{4.45\}), 4.40\left(\mathrm{t}, 1 \mathrm{H}, J=5.4 \mathrm{~Hz}, \mathrm{~S}_{1}{ }^{\alpha}\right), 4.34-4.21(\mathrm{~m}, 7$ $\mathrm{H}, \mathrm{S}_{2}{ }^{\alpha}\{4.32\}, \mathrm{P}_{1-5}{ }^{\alpha}\{4.29\}\{4.28\}\{4.28\}\{4.26\}\{4.26\}$,
$\left.\mathrm{T}_{1}^{\alpha}\{4.24\}\right), 4.20\left(\mathrm{~d}, 1 \mathrm{H}, J=4.5 \mathrm{~Hz}, \mathrm{~V}^{\alpha}\right), 4.16-4.10(\mathrm{~m}, 1 \mathrm{H}$, $\left.\mathrm{A}_{4}{ }^{\alpha}\right), 4.11-4.05\left(\mathrm{~m}, 2 \mathrm{H}, \mathrm{T}_{1}{ }^{\beta}, \mathrm{T}_{2}{ }^{\beta}\right), 4.00(\mathrm{dd}, 1 \mathrm{H}, J=3.8 \mathrm{~Hz}$, $11.1 \mathrm{~Hz}$, GalNAc-H2), 3.95-3.89 (m, $2 \mathrm{H}, \mathrm{T}_{2}{ }^{\alpha}\{3.93\}$, GalNAc$\mathrm{H} 5\{3.89\}), 3.89-3.82\left(\mathrm{~m}, 4 \mathrm{H}, \mathrm{G}_{1}{ }^{\alpha}\{3.87\}, \mathrm{G}_{1}{ }^{\alpha}\{3.84\}, \mathrm{G}_{2}{ }^{\alpha}\right.$ $\{3.84\}$, GalNAc-H4 $\{3.84\}), 3.82-3.74(\mathrm{~m}, 3 \mathrm{H}$, GalNAc-H3 $\left.\{3.80\}, \mathrm{G}_{2}{ }^{\alpha}\{3.80\}, \mathrm{S}_{1}{ }^{\beta}\{3.79\}\right), 3.77-3.74\left(\mathrm{~m}, 1 \mathrm{H}, \mathrm{S}_{1}{ }^{\beta}\right)$, $3.74-3.61\left(\mathrm{~m}, 11 \mathrm{H}, \mathrm{P}_{1-5} \delta\{3.70\}\{3.68\}\{3.68\}\{3.68\}\{3.67\}\right.$, GalNAc-H6 $\{3.65\}, 2 \times \mathrm{CH}_{2}$-spacer $\left.\{3.65\}\{3.62\}\right), 3.61-3.31$ (m, $15 \mathrm{H}, \mathrm{S}_{2}{ }^{\beta}\{3.59\}, 4 \times \mathrm{CH}_{2}$-spacer $\{3.58\}, 4 \times \mathrm{CH}_{2}$-spacer $\left.\{3.54\}, \mathrm{P}_{1-5} \delta\{3.58-3.48\}\right), 3.19\left(\mathrm{dd}, 1 \mathrm{H}, J=\mathrm{Hz}, \mathrm{H}^{\alpha}\right)$, 3.11-3.02 (m, $5 \mathrm{H}, \mathrm{CH}_{2}$-spacer $\left.\{3.09\}, \mathrm{R}^{\delta}\{3.07\}, \mathrm{H}^{\alpha}\{3.07\}\right)$, $2.85\left(\mathrm{dd}, 1 \mathrm{H}, J=6.6 \mathrm{~Hz}, 17.4 \mathrm{~Hz}, \mathrm{D}^{\beta}\right), 2.76(\mathrm{dd}, 1 \mathrm{H}, J=6.6$ $\left.\mathrm{Hz}, 17.0 \mathrm{~Hz}, \mathrm{D}^{\beta}\right), 2.69-2.38\left(\mathrm{~m}, 4 \mathrm{H}, \mathrm{CH}_{2}\right.$-spacer $\{2.62\}, \mathrm{hT}^{\alpha}$ $\{2.57\}, \mathrm{CH}_{2}$-spacer $\left.\{2.55\}, \mathrm{hT}^{\alpha}\{2.43\}\right), 2.26-2.07(\mathrm{~m}, 5 \mathrm{H}$, $\left.\mathrm{P}_{1-5} \beta\{2.20\}\{2.18\}\{2.17\}\{2.16\}\{2.14\}\right), 2.00-1.48$ (m, 23 $\mathrm{H}, \mathrm{P}_{1-5}{ }^{\gamma}\{1.98-1.80\}, \mathrm{P}_{1-5} \beta\{1.92-1.70\}, \mathrm{CH}_{3}-\mathrm{AcNH}\{1.93\}$, $\left.\mathrm{V}^{\beta}\{1.85\}, \mathrm{R}^{\beta}\{1.71\}, \mathrm{R}^{\beta}\{1.61\}, \mathrm{R}^{\gamma}\{1.54\}\right), 1.29-1.18(\mathrm{~m}, 12$ $\left.\mathrm{H}, \mathrm{A}_{1-4} 4^{\beta}\{1.26\},\{1.24\},\{1.21\},\{1.21\}\right), 1.10-1.02(\mathrm{~m}, 9 \mathrm{H}$, $\left.\mathrm{T}_{1}^{\gamma}, \mathrm{T}_{2}^{\gamma}, \mathrm{hT}^{\delta}\{1.06\},\{1.04\},\{1.04\}\right), 0.76(\mathrm{~d}, 3 \mathrm{H}, J=6.7 \mathrm{~Hz}$, $\left.\mathrm{V}^{\gamma}\right), 0.72\left(\mathrm{~d}, 3 \mathrm{H}, J=6.7 \mathrm{~Hz}, \mathrm{~V}^{\gamma}\right) ;{ }^{13} \mathrm{C}$ NMR (101 MHz, DEPT, HSQC, $\left.\mathrm{D}_{2} \mathrm{O}\right), \delta(\mathrm{ppm})=175.9,174.9,174.4,174.4,173.9$, $173.5,173.5,173.1,172.9,172.8,172.6,172.5,172.4,172.4$, 172.0, 171.5, 171.3, 171.2, 171.1, 170.9, $170.8(\mathrm{C}=\mathrm{O}), 163.1$, 162.7 (TFA), $156.7(\mathrm{C}=\mathrm{NH}), 133.4\left(\mathrm{H}^{\beta}\right), 128.4\left(\mathrm{H}^{\varepsilon}\right), 117.3$ $\left(\mathrm{H}^{\delta}\right), 98.0$ (GalNAc-C1), $76.3\left(\mathrm{hT}^{\gamma}\right), 69.6,69.5,69.5,69.4$ ( $\mathrm{CH}_{2}$-Spacer), 71.3 (GalNAc-C5), 67.4 (GalNAc-C3), 61.5 (GalNAc-C6), 61.2, $61.1\left(\mathrm{~S}_{1}{ }^{\beta}, \mathrm{S}_{2}{ }^{\beta}\right), 60.8,60.5,60.0,59.7,58.7$ $\left(\mathrm{P}_{1-5^{\alpha}}{ }^{\alpha}\right), 59.6,59.3\left(\mathrm{~T}_{1}{ }^{\alpha}, \mathrm{T}_{2}{ }^{\alpha}\right), 58.9\left(\mathrm{~V}^{\alpha}\right), 55.5\left(\mathrm{~S}_{2}{ }^{\alpha}\right), 54.9\left(\mathrm{~S}_{1}{ }^{\alpha}\right)$, $52.3\left(\mathrm{H}^{\alpha}\right), 51.1\left(\mathrm{R}^{\alpha}\right), 50.5\left(\mathrm{hT}^{\beta}\right), 50.1\left(\mathrm{D}^{\alpha}\right), 50.0$ (GalNAc-C2), $49.6\left(\mathrm{~A}_{4}{ }^{\alpha}\right), 48.0,47.9,47.7,47.4\left(\mathrm{P}_{1-5}{ }^{\delta}\right), 47.8,47.6,47.6\left(\mathrm{~A}_{1}{ }^{\alpha}\right.$, $\left.\mathrm{A}_{2}{ }^{\alpha}, \mathrm{A}_{3}{ }^{\alpha}\right), 42.4,42.3\left(\mathrm{G}_{1}{ }^{\alpha}, \mathrm{G}_{2}{ }^{\alpha}\right), 40.5\left(\mathrm{CH}_{2}\right.$-spacer $), 39.0\left(\mathrm{R}^{\delta}\right)$, $36.1\left(\mathrm{hT}^{\alpha}\right), 35.0\left(\mathrm{D}^{\beta}\right), 34.0\left(\mathrm{CH}_{2}\right.$-spacer $), 29.9\left(\mathrm{~V}^{\beta}\right), 29.6,29.3$, 29.2, 29.1, $28.7\left(\mathrm{P}_{1-5}{ }^{\beta}\right), 27.4\left(\mathrm{R}^{\beta}\right), 26.2\left(\mathrm{H}^{\beta}\right), 24.7,24.6,24.5$, $24.3\left(\mathrm{P}_{1-5}{ }^{\gamma}\right), 23.9\left(\mathrm{R}^{\gamma}\right), 21.9\left(\mathrm{CH}_{3}-\mathrm{AcNH}\right), 18.8,18.7\left(\mathrm{~T}_{1}^{\gamma}, \mathrm{T}_{2}^{\gamma}\right)$, 18.3, $17.7\left(\mathrm{~V}^{\gamma \alpha}, \mathrm{V}^{\gamma \beta}\right), 16.6\left(\mathrm{hT}^{\delta}\right), 16.2,15.4,15.1,15.0\left(\mathrm{~A}_{1-4}{ }^{\beta}\right)$; HR-ESI-MS: $1154.0817\left([\mathrm{M}+2 \mathrm{H}]^{2+}\right.$, calc.: 1154.0774).

\section{Supporting Information}

\section{Supporting Information File 1}

NMR spectra of compounds 2a, 2b, 4, 7, 8 and HPLC chromatogram of compound $\mathbf{2 a}$.

[http://www.beilstein-journals.org/bjoc/content/ supplementary/1860-5397-6-47-S1.pdf]

\section{Acknowledgements}

This work was supported by the Deutsche Forschungsgemeinschaft (Emmy Noether Fellowship to A. H.-R.). We thank Prof. Dr. H. Kunz for fruitful discussions. 


\section{References}

1. Dwek, R. A. Chem. Rev. 1996, 96, 683-720. doi:10.1021/cr940283b

2. Lis, H.; Sharon, N. Eur. J. Biochem. 1993, 218, 1-27. doi:10.1111/j.1432-1033.1993.tb18347.x

3. Varki, A. Glycobiology 1993, 3, 97-130. doi:10.1093/glycob/3.2.97

4. Pratt, M. R.; Bertozzi, C. R. Chem. Soc. Rev. 2005, 34, 58-68. doi:10.1039/b400593g

5. Taylor-Papadimitriou, J.; Epenetos, A. A. Trends Biotechnol. 1994, 12, 227-233. doi:10.1016/0167-7799(94)90121-X

6. Danishefsky, S. J.; Allen, J. R. Angew. Chem., Int. Ed. 2000, 39, 836-863.

doi:10.1002/(SICI)1521-3773(20000303)39:5<836::AID-ANIE836>3.0. CO;2-I

7. Slovin, S. S.; Keding, S. J.; Ragupathi, G. Immunol. Cell Biol. 2005, 83, 418-428. doi:10.1111/j.1440-1711.2005.01350.x

8. Becker, T.; Dziadek, S.; Wittrock, S.; Kunz, H. Curr. Opin. Mol. Ther. 2007, 9, 35-44. doi:10.2174/156800906778194577

9. Liakatos, A.; Kunz, H. Curr. Opin. Mol. Ther. 2007, 9, 35-44.

10. Buskas, T.; Thompson, P.; Boons, G.-J. Chem. Commun. 2009, 5335-5349. doi:10.1039/b908664c

11. Urban, D.; Skrydstrup, T.; Beau, J.-M. Chem. Commun. 1998, 955-956. doi:10.1039/a801196f

12. Röhrig, C. H.; Takhi, M.; Schmidt, R. R. Synlett 2001, 1170-1172. doi:10.1055/s-2001-15144

13. Kuberan, B.; Sikkander, S. A.; Tomiyama, H.; Linhardt, R. J. Angew. Chem., Int. Ed. 2003, 42, 2073-2075. doi:10.1002/anie.200351099

14. Cipolla, L.; Rescigno, M.; Leone, A.; Peri, F.; La Ferla, B.; Nicotra, F. Bioorg. Med. Chem. 2002, 10, 1639-1646. doi:10.1016/S0968-0896(01)00433-3

15. Rich, J. J.; Bundle, D. R. Org. Lett. 2004, 6, 897-900. doi:10.1021/ol036460p

16. Bousquet, E.; Spadaro, A.; Pappalardo, M. S.; Bernardini, R.; Romeo, R.; Panza, L.; Ronsisvalle, G. J. Carbohydr. Chem. 2000, 19, 527-541. doi:10.1080/07328300008544097

17. Bundle, D. R.; Rich, J. J.; Jacques, S.; Yu, H. N.; Nitz, M.; Ling, C.-C. Angew. Chem., Int. Ed. 2005, 44, 7725-7729. doi:10.1002/anie.200502179

18. Rich, J. J.; Wakarchuk, W. W.; Bundle, D. R. Chem.-Eur. J. 2006, 12, 845-858. doi:10.1002/chem.200500518

19. Wu, X.; Lipinski, T.; Paszkiewicz, E.; Bundle, D. R. Chem.-Eur. J. 2008, 14, 6474-6482. doi:10.1002/chem.200800352

20. Mersch, C.; Wagner, S.; Hoffmann-Röder, A. Synlett 2009, 2167-2171. doi:10.1055/s-0029-1217566

21. Hook, D. F.; Bindschädler, P.; Mahajan, Y. R.; Šebesta, R.; Kast, P.; Seebach, D. Chem. Biodiversity 2005, 2, 591-632. doi:10.1002/cbdv.200590039

22. Aguilar, M.-I.; Purcell, A. W.; Devi, R.; Lew, R.; Rossjohn, J.; Smith, A. I.; Perlmutter, P. Org. Biomol. Chem. 2007, 5, 2884-2890. doi:10.1039/b708507a

23. Cheng, R. P.; Gellman, S. H.; DeGrado, W. F. Chem. Rev. 2001, 101, 3219-3232. doi:10.1021/cr000045i

24. Steer, D. L.; Lew, R. A.; Perlmutter, P.; Smith, A. I.; Aguilar, M.-I. Curr. Med. Chem. 2002, 9, 811-822.

25. Seebach, D.; Gardiner, J. Acc. Chem. Res. 2008, 41, 1366-1375. doi:10.1021/ar700263g

26. Saludes, J. P.; Ames, J. B.; Gervay-Hague, J. J. Am. Chem. Soc. 2009, 131, 5495-5505. doi:10.1021/ja808286x

27. Disney, M. D.; Hook, D. F.; Namoto, K.; Seeberger, P. H.; Seebach, D. Chem. Biodiversity 2005, 2, 1624-1634. doi:10.1002/cbdv.200590132
28. Norgren, A. S.; Geitmann, M.; Danielson, U. H.; Arvidsson, P. I. J. Mol. Recognit. 2007, 20, 132-138. doi:10.1002/jmr.821

29. Norgren, A. S.; Norberg, T.; Arvidsson, P. I. J. Pept. Sci. 2007, 13, 717-727. doi:10.1002/psc.832

30. Norgren, A. S.; Arvidsson, P. I. Org. Biomol. Chem. 2005, 3, 1359-1361. doi:10.1039/b503237g

31. Norgren, A. S.; Arvidsson, P. I. J. Org. Chem. 2008, 73, 5272-5278. doi:10.1021/j08003265

32. Paulsen, H.; Hölck, J.-P. Carbohydr. Res. 1982, 109, 89-107. doi:10.1016/0008-6215(82)84033-0

33. Brocke, C.; Kunz, H. Synthesis 2004, 525-542. doi:10.1055/s-2004-815962

34. Dziadek, S.; Brocke, C.; Kunz, H. Chem.-Eur. J. 2004, 10, 4150-4162. doi:10.1002/chem.200400228

35. Dziadek, S.; Kowalczyk, D.; Kunz, H. Angew. Chem., Int. Ed. 2005, 44, 7624-7630. doi:10.1002/anie.200501593

36. Kaiser, A.; Gaidzik, N.; Westerlind, U.; Kowalczyk, D.; Hobel, A.; Schmitt, E.; Kunz, H. Angew. Chem., Int. Ed. 2009, 48, 7551-7555. doi:10.1002/anie.200902564

37. Westerlind, U.; Schröder, H.; Hobel, A.; Gaidzik, N.; Kaiser, A.; Niemeyer, C. M.; Schmitt, E.; Waldmann, H.; Kunz, H. Angew. Chem., Int. Ed. 2009, 48, 8263-8267. doi:10.1002/anie.200902963

38. Fréchet, J. M. J.; Haque, K. E. Tetrahedron Lett. 1975, 16, 3055-3056. doi:10.1016/S0040-4039(00)75071-4

39. Dourtoglou, V.; Gross, B.; Lambropoulou, V.; Zioudrou, C. Synthesis 1984, 572-574. doi:10.1055/s-1984-30895

40. Carpino, L. A. J. Am. Chem. Soc. 1993, 115, 4397-4398. doi:10.1021/ja00063a082

41. Keil, S.; Claus, C.; Dippold, W.; Kunz, H. Angew. Chem., Int. Ed. 2001, 40, 366-369.

doi:10.1002/1521-3773(20010119)40:2<366::AID-ANIE366>3.0.CO;2-J

42. Lombardi, P. Chem. Ind. (London) 1994, 708.

\section{License and Terms}

This is an Open Access article under the terms of the Creative Commons Attribution License (http://creativecommons.org/licenses/by/2.0), which permits unrestricted use, distribution, and reproduction in any medium, provided the original work is properly cited.

The license is subject to the Beilstein Journal of Organic Chemistry terms and conditions:

(http://www.beilstein-journals.org/bjoc)

The definitive version of this article is the electronic one which can be found at: $\underline{\text { doi: } 10.3762 / \text { bjoc. } 6.47}$ 